\title{
Septal Schwannoma of the Nose: A Rare Case
}

\author{
Case Report $>\begin{aligned} & \text { Manish Gupta, Naiya Rao, Chandpreet Kour, Ivreet Kaur } \\ & \text { Maharishi Markandeshwar Institute of Medical Sciences and Research, Ambala, India }\end{aligned}$
}

Abstract

A schwannoma is a benign tumor arising from Schwann cells present in the nerve sheath of myelinated nerves. It is rarely seen in the nose and paranasal sinuses, and it very rarely originates from the nasal septum. A 40-year-old female presented to our outpatient department with a gradual onset, progressive, left nasal obstruction since the last 4 years. On performing a local examination, a soft tissue mass occupying the left nasal cavity was

\section{Introduction}

A schwannoma is a benign tumor is only seen arising from the nerve sheath; thus, it isonlyseen in myelinated nerves. In the head and neck region, the most common site is the eighth cranial nerve (vestibulocochlear); other sites include the scalp, face, parotid gland, oral cavity, pharynx, larynx, and trachea (1). Only $4 \%$ of schwannomas seen in the head and neck region arise from the nose and paranasal sinuses and they may arise from branches of the trigeminal nerve (ophthalmic or maxillary) or from the autonomic nervous system (1). They do not arise from optic or olfactory nerves as these nerves lack sheaths that contain Schwann cells (1). The diagnosis of a nasal schwannoma is usually only histopathological due to the lack of radiological characteristic features (2).

\section{Case Presentation}

A 40-year-old female presented with a gradual onset, progressive, left nasal obstruction sincethe last 4 years. There was a history of an occasional mucoid, nasal discharge, and bleeding. There was no history of any trauma, irra- seen. Surgery via the external approach was performed; the mass was found to arise from the nasal septum and was totally excised. Histopathologic examination revealed a schwannoma. Nasal septal schwannoma along with review of the literature is a rare entity. It should be considered in the differential diagnosis of benign tumors of the nasal cavity.

Keywords: Schwannoma, nasal septum, lateral rhinotomy

diation, or surgery, and the family history was insignificant. An external examination of the nose showed expansion of the left nasal bone and the frontal process of the maxilla. Anterior rhinoscopy revealed a single, large, pale, smooth, nontender, firm, and soft tissue mass occupying the entire left nasal cavity with no turbinate visible (Figure 1). A probe could be passed lateral, superior, and inferior, but not medial, to the swelling. There was no bleeding on probing and it was slightly mobile anteroposteriorly. On performing a cold spatula test, fogging was completely absent on the left side. The mass was not seen on posterior rhinoscopy, and there was no palatal bulge. The rest of the otorhinolaryngological, head and neck, and general physical examinations was unremarkable.

Contrast-enhanced computed tomography showed a soft tissue mass (39-45 HU) completely occupyingthe anterior half of the left nasal cavity and reaching external nares with heterogeneous enhancement on postcontrast scans (Figure 2). The orbit and none of the paranasal sinuses were involved, and there was no evidence of bone destruction or intracranial 
extension. All routine blood and urine investigation results were normal.

Informed consent was taken from the patient. The patient underwent surgical excision of the mass via the external approach (lateral rhinotomy) under general anesthesia (Figure 3 ) as the mass was considered to be benign because of the clinical and radiological properties. Intraoperative endoscopy revealed that the mass arose from the anterior nasal septum. The cuff of the surrounding mucoperichondrium at the site of attachment over the cartilaginous septum was taken along with the en bloc excised mass. Hemostasis was achieved with bipolar cautery. A medicated ribbon gauze was packed in the left nasal cavity and was removed after 48 hours. The postoperative phase was uneventful.

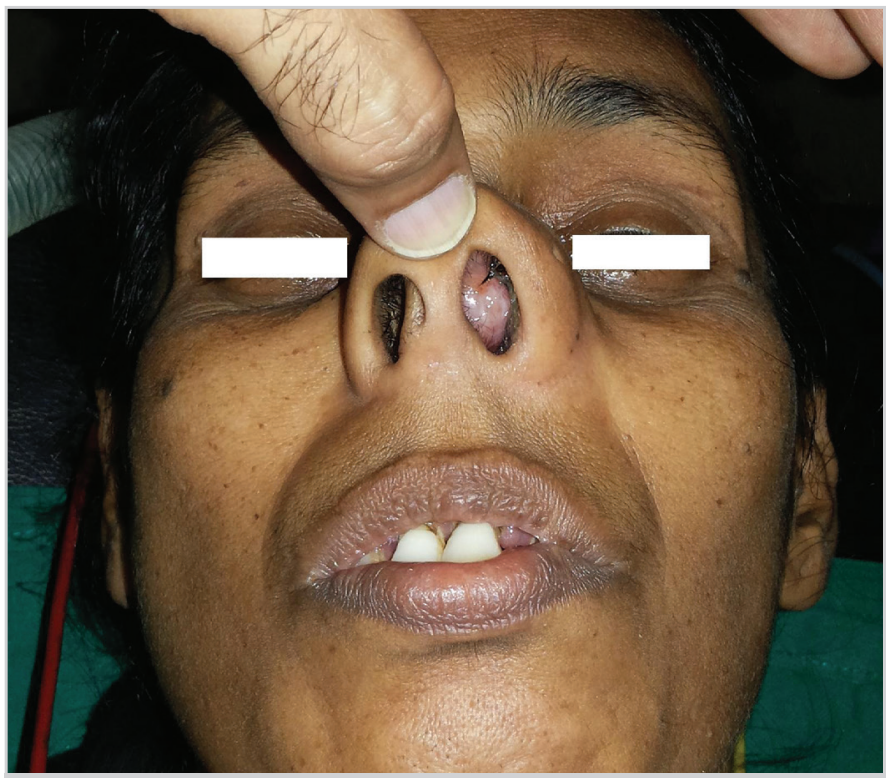

Figure 1. Clinical picture showing a single, large, pale, smooth, soft tissue mass occupying the entire left nasal cavity

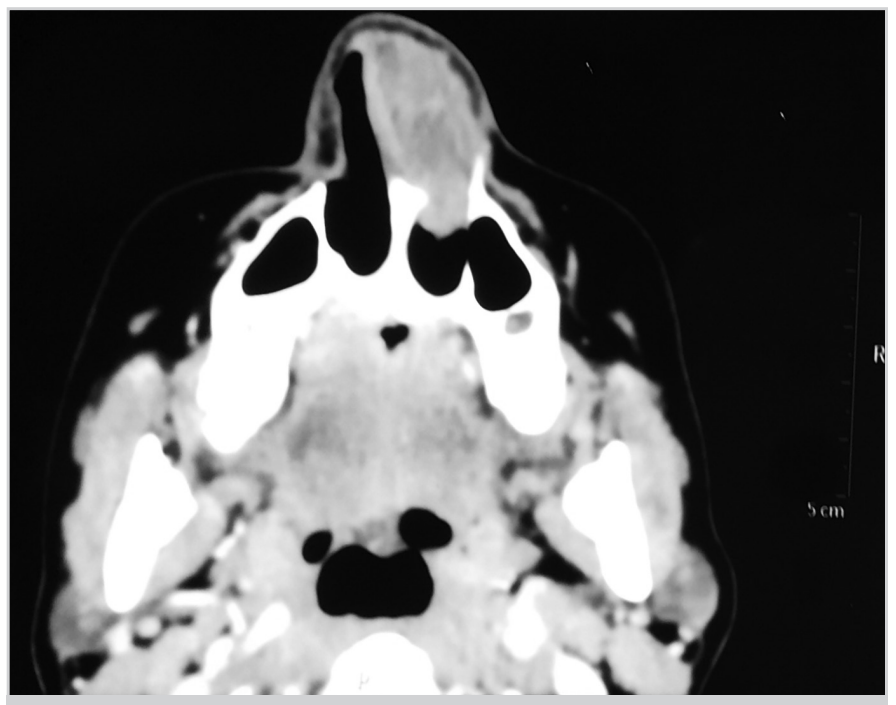

Figure 2. Contrast-enhanced computed tomography finding of the nose and paranasal sinuses; the axial sectionshowed a soft tissue mass completely occupying the anterior half of the left nasal cavity with heterogeneous enhancement
Histopathologic examination showed the presence of respiratory epithelium with the absence of capsules (Figure 4). A high-power examination showed a tumor with spindle cells arranged in the Antoni A pattern and Verocay bodies, suggesting a schwannoma.

In the one year follow-up, the septal mucoperichondrial defect had re-epithelialized, and there was no recurrence.

\section{Discussion}

The literature mentions approximately only 70 cases of nose and paranasal sinus schwannomas that are mostly seen in adults aged 40-60 years and without gender or racial predilection (3). The most commonly involved sinus is the ethmoid sinus, followed by the maxillary sinus, nasal cavity,

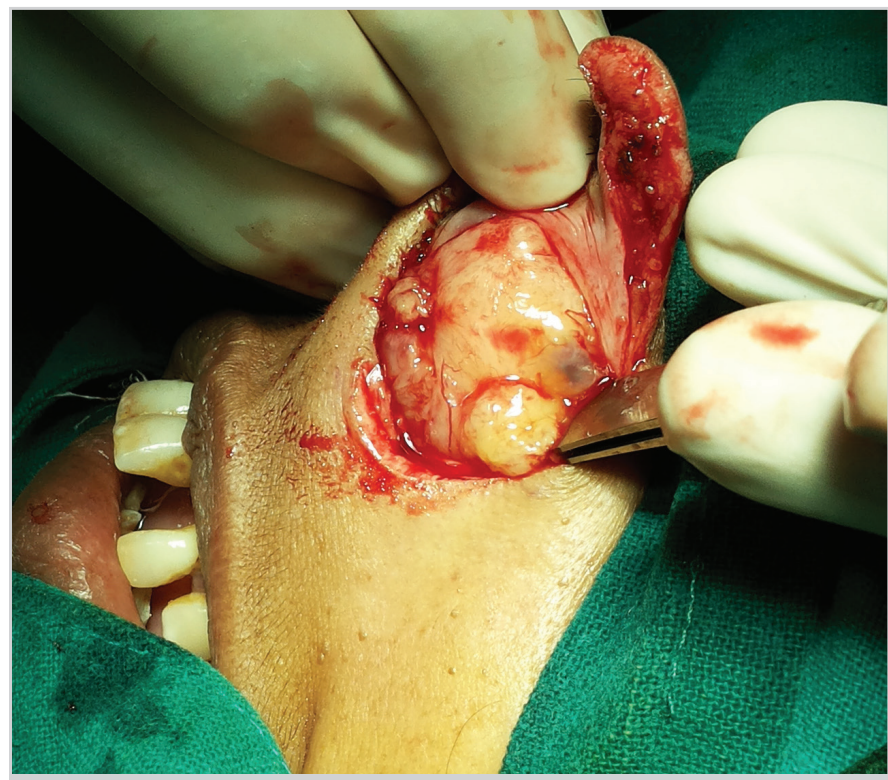

Figure 3. The lateral rhinotomy incision, elevated flap, and mass occupying the nasal cavity with an attachment on the septum

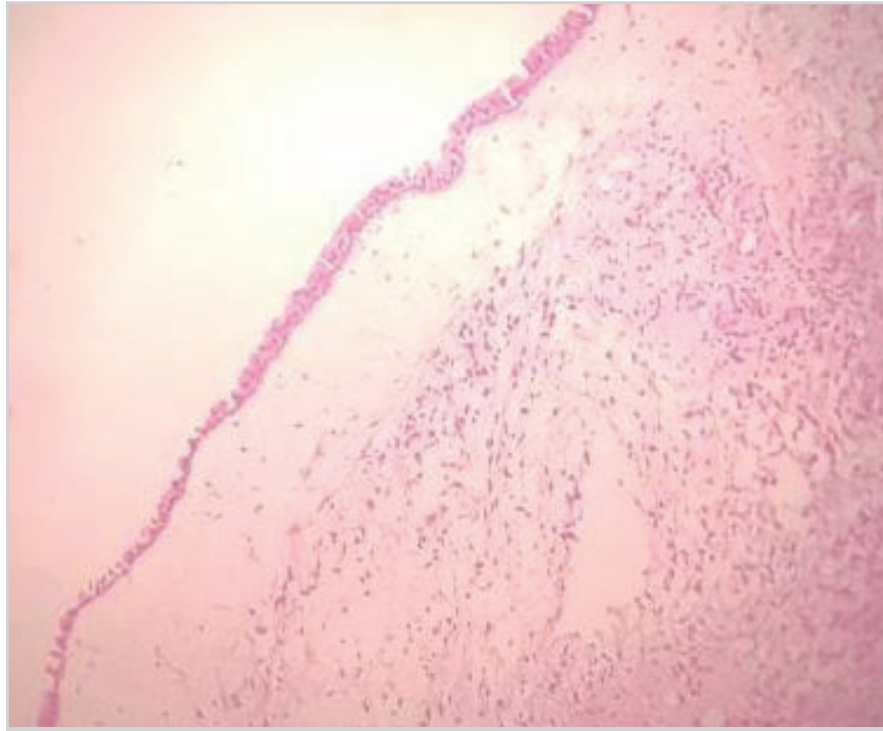

Figure 4. Low-power (10X), H\&E stained microscopic slide picture showing the presence of hyperplastic respiratory epithelium with the absence of a capsule 
and sphenoid (4). Those arising from the nasal septum are even rarer; only 11 cases have been reported in the literature (4). Being slow-growing tumors, they mostly remain asymptomatic, till they attain a large size. A nasal septal schwannoma may present with nasal blockage, discharge, anosmia, a deformity of the nasal pyramid, headache, or epistaxis (5). Involvement of the paranasal sinuses and orbit may lead to facial swelling and proptosis. It may present with trans-septal extension and opposite nasal cavity involvement (6).It rarely undergoes malignant transformation (6). As reported in the literature, the nerve of origin cannot be identified, and no neurological symptom or postoperative deficit is seen, which was similar to that seen in our case (7).

The differential diagnosis for the nasal mass includes the following: antrochoanal polyp, concha bullosa, lymphoma, inverted papilloma, capillary hemangioma, fibrous dysplasia, mucocoele, meningioma, neurofibroma, neurilemmoma, enchondroma, septal dermoid, melanoma, idiopathic midline granuloma, myxoma, fibromyxoma, squamous cell carcinoma, chondrosarcoma, and intranasal extension of the nasopharyngeal angiofibroma, esthesioneuroblastoma, and malignant neurilemmoma (7-9). Due to this variety of possible pathologies, it is difficult to make a diagnosis based on clinical results. Even contrast-enhanced computed tomography findings are not specific for diagnosing schwannomas, but they are helpful in evaluating the site of origin and extent of tumor. Biopsy may be considered when in doubt to diagnose cases of malignancy. As our patient's tumor looked to be benign on performing a clinical examination and radiological investigation, instead of preoperative punch biopsy, the patient was directly taken for excisional biopsy, which was curative.

Schwannomas are often encapsulated, well circumscribed, and connected to the nerve tissue. Their histopathology exhibits Antoni $\mathrm{A}$ and Antoni $\mathrm{B}$ patterns in varying proportions. The Antoni A pattern comprises organized compact stroma with spindle cells and parallel rows of palisading nuclei. The Antoni B pattern has disorganized loose myxoid stroma with few spindle cells. Nasal schwannomas are different from schwannomas arising from other sites in their histopathology in two ways (10). They do not have fibrous encapsulation and have hypercellularity. As autonomic nerve fibers innervating the nasal mucosa are devoid of perineural sheaths, schwannomas arising from this region are noncapsulated (6). Similarly, in our patient, no capsule was noted on the tumor surface, and no typical Antoni B pattern was noted.

Excision by lateral rhinotomy or endoscopic endonasal surgery is the treatment of choice for schwannomas, and postoperative recurrences are rare (5). In our patient, due to the tumor size and limited endoscope entry into the nasal cavity, the lateral approach was adopted to remove the tumor en bloc. The possibility of a schwannoma should be considered when a soft tissue mass is encountered in the nasal cavity.

\section{Conclusion}

Schwannomas should be considered in the differential diagnosis whenever a benign-looking mass is seen inside the nasal cavity.

Informed Consent: Written informed consent was obtained from patient who participated in this study.

Peer-review: Externally peer-reviewed.

Author Contributions: Concept - M.G.; Design - M.G.; Supervision - M.G., C.K.; Data Collection and/or Processing - M.G., I.K.; Analysis and/or Interpretation - M.G., I.K.; Literature Search - M.G., N.R.; Writing - M.G., N.R.; Critical Reviews - M.G., N.R., C.K., I.K.

Conflict of Interest: No conflict of interest was declared by the authors.

Financial Disclosure: The authors declared that this study has received no financial support.

\section{References}

1. Gulia JS, Yadav SS, Basur SK, Hooda A. Schwannoma of the membranous nasal septum. Braz J Otorhinolaryngol 2013; 79: 789. [CrossRef]

2. Yu E, Mikulis D, Nag S. CT and MR imaging findings in sinonasal schwannoma. Am J Neuro Radiol 2006; 27: 929-30.

3. Pauna HF, Carvalho GM, Guimaraes AC, Maunsell RC, Sakano E. Schwannoma of the nasal septum: evaluation of unilateral nasal mass. Braz J Otorhinolaryngol 2013; 79: 403. [CrossRef]

4. Calceterra TC, Rich JR, Ward PW. Neurilemoma of the sphenoid sinus. ArchOtolaryngol 1974; 100: 383-85. [CrossRef]

5. Mey KH, Buchwald C, Daugaard S, Prause JU. Sinonasal schwannoma- a clinicopathological analysis of five rare cases. Rhinology 2006; 44: 46-52.

6. Pasic TR, Makielski K. Nasal schwannoma. Otolaryngol Head Neck Surg 1990; 103: 943-6. [CrossRef]

7. Kim YS, Kim HJ, Kim CH, Kim J. CT and MR findings of sinonasal schwannoma: a review of 12 cases. Am J Neuro Radiol 2013; 34: 628-33. [CrossRef]

8. Liu YP, Huang JK, Cheng SJ, Sheu CY. Neurilemmoma of the nasal septum: A case report. Chin J Radiol 2002; 27: 79-82.

9. Valencia MP, Castillo M. Congenital and acquired lesions of the nasal septum: a practical guide for differential diagnosis. Radiographics 2008; 28: 205-24. [CrossRef]

10. Hasegawa SL, Mentzel T, Fletcher CD. Schwannomas of the sinonasal tract and nasopharynx. Mod Pathol 1997; 10: 777-84. 